\title{
Consulta externa en clínicas veterinarias de Manizales (Colombia): análisis epidemiológico en caninos
}

Jefferson Muñoz-Pérez ${ }^{\star}, \mathrm{MVZ}_{1,2}$, Ginés Fernando Ramírez-Benavides, M.Sc. ${ }_{1}$ Luisa Fernanda Garcés-Mendoza, $\mathrm{MVZ}_{1}$

${ }_{1}$ Universidad de Caldas, Facultad de Ciencias Agropecuarias, Departamento de Salud Animal, Manizales, Colombia

${ }_{2}$ Clínica Veterinaria El Poblado, Medellín, Colombia

Recibido: 4 de junio del 2015

Aprobado: 16 de octubre del 2015

*Autor de correspondencia: Jefferson Muñoz Pérez, Universidad de Caldas, Facultad de Ciencias Agropecuarias, Departamento de Salud animal. Calle 65 n. ${ }^{\circ} 26-10$, Manizales, Caldas, Colombia. Teléfono: (576) 8861250. Correo electrónico: jeff.mp@outlook.com

Cómo citar este artículo: Muñoz-Pérez J, Ramírez-Benavides GF, Garcés-Mendoza LF. Consulta externa en clínicas veterinarias de Manizales (Colombia): análisis epidemiológico en caninos. Spei Domus. 2015;11(23):17-23. doi: http://dx.doi. org/10.16925/sp.v11i23.1364

Resumen. Introducción: la importancia de las mascotas y su relación directa con la salud pública y las zoonosis influyen notablemente en la necesidad de contar con bases de datos epidemiológicas en Colombia. Materiales y métodos: durante ocho meses, se obtuvo información de caninos atendidos en nueve clínicas veterinarias de Manizales, Colombia. Fueron catalogadas variables como raza, sexo, estado reproductivo, motivo de consulta, hallazgos clínicos y diagnósticos presuntivos. Se clasificó a la población en cuatro grupos de edades: Grupo A (0-3 meses); Grupo B (4-12 meses); Grupo C (13-36 meses); Grupo D (>37 meses). Se evaluaron las relaciones directas entre variables y se analizó la existencia de diferencias significativas entre grupos y datos estadísticos relevantes. Resultados: se registraron 2414 caninos, 1381 machos (11,3\% castrados), 1033 hembras (26\% esterilizadas). La distribución de población en cada subgrupo fue: Grupo A (10,2\%); Grupo в (24,7\%); Grupo C (23,6\%); Grupo D (41,5\%). Se encontró diferencia significativa $(\mathrm{p}<0,05)$ entre los subgrupos y la prevalencia de afección de algunos sistemas. El sistema digestivo fue el motivo de consulta con mayor frecuencia en el Grupo A (52,7\%); seguido del Grupo в (34,1\%). El principal motivo de consulta en todos los grupos fue por afección del sistema digestivo con 30,07\%; las dermopatías con 26,39\% fueron el segundo motivo de consulta más frecuente. Conclusiones: la frecuencia de aparición de las diferentes afecciones concuerda con investigaciones similares y estudios epidemiológicos en otras ciudades del país y del mundo. Se recomienda que en otras ciudades del país se aporten investigaciones similares.

Palabras claves: epidemiología, Manizales, consulta externa, clínica veterinaria. 


\title{
Outpatient Service at Veterinary Clinics in Manizales (Colombia): Epidemiological Analysis in Dogs
}

\begin{abstract}
Introduction: The importance of pets and their direct relationship with public health and zoonoses greatly influence the need for epidemiological databases in Colombia. Materials and methods: For eight months, information of dogs cared for at nine veterinary clinics in Manizales, Colombia was obtained. Variables such as race, sex, reproductive status, reason for consultation, clinical findings, and presumptive diagnoses were cataloged. Population was classified into four age groups: Group A (0-3 months); Group в (4-12 months); Group C (13-36 months); Group D ( $>37$ months). Direct relationships among variables were evaluated and the existence of significant differences between groups and relevant statistical data was analyzed. Results: 2414 dogs, 1381 males (11.3\% castrated), 1033 females (26\% sterilized) were reported. The distribution of population in each subgroup was: Group A (10.2\%); Group в (24.7\%); Group с (23.6\%); Group D (41.5\%). A significant difference $(\mathrm{p}<0.05)$ was found between subgroups and the prevalence of conditions in some systems. The digestive system was the most frequent reason for consultation in Group A (52.7\%), followed by Group в (34.1\%). The main reason for consultation in all groups was by conditions of the digestive system (30.07\%); skin diseases $(26.39 \%)$ were the second most frequent reason for consultation. Conclusions: Frequency of occurrence of different conditions is consistent with similar research and epidemiological studies in other cities of the country and the world. It is recommended that other cities conduct similar research.
\end{abstract}

Keywords: epidemiology, Manizales, outpatient service, veterinary clinic.

\section{Consulta externa em clínicas veterinárias de Manizales (Colômbia): análise epidemiológica em cães}

Resumo. Introdução: a importância dos animais de estimação e sua relação direta com a saúde pública e a zoonose influenciam notavelmente na necessidade de contar com bases de dados epidemiológicas na Colômbia. Materiais e métodos: por oito meses, obteve-se informação de caninos atendidos em nove clínicas veterinárias de Manizales (Colômbia). Foram catalogadas variáveis como raça, sexo, estado reprodutivo, motivo de consulta, achados clínicos e diagnósticos presumíveis. Classificou-se a população em quatro faixas etárias: Grupo А (0-3 meses); Grupo в (4-12 meses); Grupo с (13-36 meses); Grupo D (>37 meses). Avaliaram-se as relações diretas entre variáveis e analisou-se a existência de diferenças significativas entre grupos e dados estatísticos relevantes. Resultados: registraram-se 2414 caninos, 1381 machos (11,3\% castrados), 1033 fêmeas (26\% esterilizadas). A distribuição de população em cada subgrupo foi: Grupo A (10,2\%); Grupo в (24,7\%); Grupo C (23,6\%); Grupo D (41,5\%). Encontraram-se diferenças significativas $(p<0,05)$ entre os subgrupos e a prevalência de afecção de alguns sistemas. O sistema digestivo foi o motivo de consulta com maior frequência no Grupo A (52,7\%); seguido do Grupo в (34,1\%). O principal motivo de consulta em todos os grupos foi por afecção do sistema digestivo com $30,07 \%$; as dermatoses com $26,39 \%$ foram o segundo motivo de consulta mais frequente. Conclusões: a frequência de ocorrência das diferentes afecções concorda com pesquisas semelhantes e estudos epidemiológicos em outras cidades do país e do mundo. Recomenda-se que em outras cidades do país se realizem pesquisas similares.

Palavras-chave: epidemiologia, Manizales, consulta externa, clínica veterinária. 


\section{Introducción}

En los últimos años, se ha observado una evolución importante del papel de los caninos en los hogares, pues se han convertido en nuevos integrantes de las familias [1]. En un estudio en el que se hizo una encuesta a nuevos propietarios de caninos, describieron sus mascotas como "hijos peludos" y tenían una percepción de relación familiar con su perro [2].

Igualmente, los perros mejoran la percepción de seguridad y el bienestar físico y mental de las personas [3]; son de ayuda terapéutica en tratamientos médicos [4], y mediante el grado de vinculación se mejora la calidad de vida de las personas y sus mascotas [5]. La tenencia de mascotas o simplemente la presencia de un animal de compañía puede tener un efecto positivo en el estatus de salud fisiológica y mental de las personas [6].

Por lo tanto, la importancia de las mascotas y su relación directa con la salud pública influyen en la necesidad de contar con bases de datos epidemiológicas de las ciudades del país como ayuda en la práctica clínica de animales de compañía, especialmente para orientar los diagnósticos e implementar planes efectivos de prevención, control de enfermedades y conocimiento de las zoonosis existentes [5]. Los objetivos de este estudio fueron: conocer la frecuencia de los motivos de consulta de propietarios de caninos atendidos en las principales clínicas veterinarias de Manizales, y conocer el sistema orgánico más afectado y establecer datos epidemiológicos útiles en la práctica clínica diaria.

\section{Materiales y métodos}

Este estudio epidemiológico descriptivo duró ocho meses consecutivos. La herramienta utilizada fue un formulario de encuesta para médicos veterinarios, pasantes o técnicos veterinarios que lo completaban al momento de diligenciar la historia clínica de cada paciente, en nueve clínicas veterinarias de Manizales. Para la elección de las unidades de análisis, se tuvieron en cuenta los siguientes criterios: distribución geográfica en toda la ciudad, prestación de servicio de laboratorio clínico, y reconocimiento y trayectoria profesional; esta información se obtuvo durante la visita a cada clínica.

Las variables seleccionadas fueron: raza, sexo, estado reproductivo, edad, tipo de alimentación, estado de desparasitación, sistema orgánico afectado (motivo de consulta), hallazgos clínicos y diagnósticos presuntivos. En las clínicas veterinarias seleccionadas, se distribuyeron carpetas con las encuestas y los consentimientos informados. Se clasificó a la población según la edad en cuatro grupos: Grupo A (0-3 meses), Grupo в (4-12 meses), Grupo с (13-36 meses) y Grupo D (37 meses en adelante).

Métodos de inclusión. Los pacientes incluidos en el estudio fueron caninos de cualquier edad, raza o sexo llevados a consulta por presentar signos clínicos de enfermedad, para cumplir con el plan vacunal o para una revisión clínica general de control.

Análisis de datos. Los datos derivados de las encuestas fueron tabulados en dos bases de datos creadas en el programa Microsoft Excel $2010^{\circ}$ : la primera contenía los datos de cada paciente: raza, edad en meses, sexo, esterilización o no, tipo de alimentación, estado de desparasitación, el sistema orgánico afectado (motivo de consulta) y los diagnósticos presuntivos dados por el médico veterinario; la segunda contenía los hallazgos clínicos observados. Cada base de datos fue analizada estadísticamente en el programa informático SAs Statistical Analysis System

Se hicieron dos análisis. El primero fue de las relaciones directas entre variables, asociando la influencia de una variable sobre otra: la afección de cada sistema orgánico con el mes de la consulta, la edad, el sexo, la raza, el estado reproductivo, el tipo de alimentación y el estado de desparasitación del paciente; también se determinó si había diferencias significativas entre ellas. El segundo análisis fue de frecuencias y porcentajes de cada variable, tanto individualmente como su representación en el total de la población, a fin de determinar la prevalencia de cada una de ellas.

\section{Resultados}

Durante el periodo analizado (octubre del 2014 a mayo del 2015), fueron registrados 2414 caninos en nueve clínicas veterinarias de Manizales. No hubo relación entre la cantidad de pacientes atendidos en cada mes y el mes de la consulta. Se obtuvo un promedio de 345 pacientes atendidos mensualmente. En total, hubo 1381 machos y 1033 hembras. Había un $11,3 \%$ de los machos castrados y un $26 \%$ de las hembras esterilizadas (ver figura 1). 
La distribución de la población estudiada según la edad fue: Grupo A (0-3 meses) 10,2\%; Grupo в (4-12 meses) 24,7\%; Grupo C (13-36 meses) 23,6\%; y Grupo D (37 meses en adelante) 41,5\%. La mayoría de individuos tenía más de 3 años (ver figura 2). Hubo diferencia significativa $(\mathrm{p}<0,05)$ entre los grupos de edad y la prevalencia de la afección de algunos sistemas. Los pacientes incluidos en este trabajo fueron caninos de 67 razas diferentes (las más frecuentes en la ciudad): mestizos, poodle, schnawzer, labrador retriever, dóberman, pincher, beagle y pug.

Problemas de salud relacionados con el sistema digestivo fue el motivo de consulta de mayor frecuencia en el Grupo A (52,7\% del grupo) y en el Grupo в $(34,1 \%)$. Los grupos restantes no tenían datos estadísticos importantes. En general, el principal motivo de consulta en todos los grupos fue por afección del sistema digestivo con un 30,07\% (ver figura 3). De los 746 perros llevados a consulta por afecciones en el sistema digestivo, 22,5\% estaba desparasitado. El $57,2 \%$ de la población total también estaba desparasitado. La mayor parte de los 129 perros estudiados tenía entre 0 y 12 meses de edad. En 656 pacientes, se hallaron 26 diagnósticos presuntivos; se destacan la gastroenteritis $(62,2 \%)$, la gastritis $(10,7 \%)$, la enfermedad periodontal $(8,84 \%)$, la indigestión $(6,09 \%)$ y la obstrucción intestinal (2,4\%).
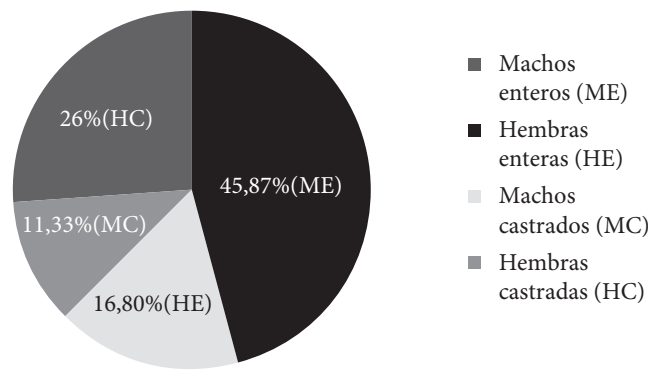

Figura 1. Estado reproductivo de la población Fuente: elaboración propia

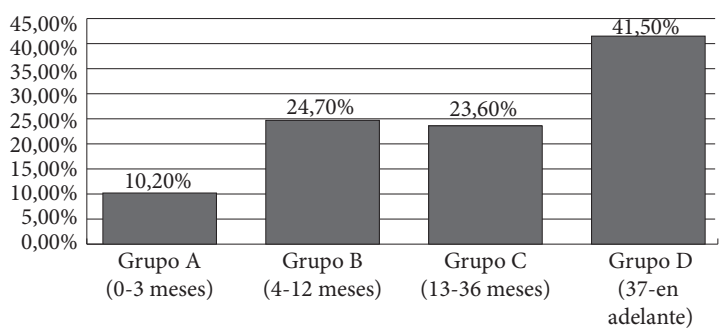

Figura 2. Distribución de la población por edad Fuente: elaboración propia

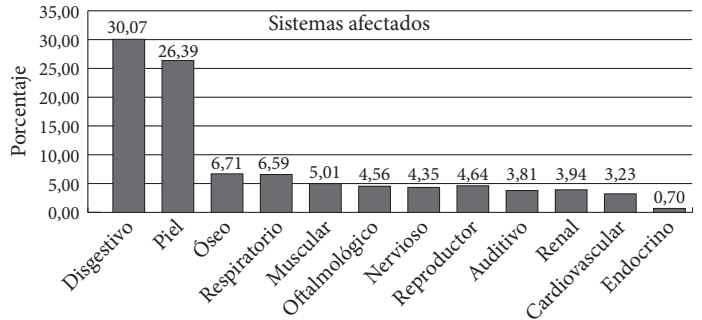

Figura 3. Frecuencia de afección por sistemas

Fuente: elaboración propia

Las dermatopatías tuvieron prevalencia en el Grupo c (31,9\% de 182 caninos) y en el Grupo в (28,2\% de 168 caninos). En los grupos restantes, no se obtuvieron datos estadísticos significativos. En general, las alteraciones de la piel (26,39\%) son el segundo motivo de consulta más frecuente en todos los grupos.

El $60 \%$ de los 657 caninos con alteraciones de la piel tenía entre 13 y 36 meses de edad y el $84,5 \%$ consumía alimento balanceado comercial, lo cual permite descartar la alimentación de origen casero como una de las causas de dermatopatías. En 462 pacientes, se encontraron 26 diagnósticos presuntivos, entre los que sobresale la dermatitis de etiología desconocida (21,6\%). Se debe tener presente que al momento de la encuesta, no se habían hecho pruebas paraclínicas para confirmar o descartar esos hallazgos en la gran mayoría de pacientes, y los diagnósticos presuntivos estaban basados en la historia clínica y el examen físico.

Las afecciones diagnosticadas fueron: dermatitis alérgica por picadura de pulga (16,2\%), demodicosis $(11,7 \%)$, pioderma $(11,7 \%)$ y dermatofitosis $(9,7 \%)$. Los demás sistemas no superaron el $9 \%$ de frecuencia de consulta y el menos afectado fue el sistema endocrino $(0,7 \%)$. Los motivos de consulta por afecciones musculares, nerviosas, oftalmológicas, de oído, respiratorias y endocrinas no tuvieron representación significativa por grupo específico de edad.

El sistema renal estuvo afectado en 84 pacientes $(3,94 \%)$. En el Grupo D, la mayoría de pacientes presentó afecciones renales (5,8\% de los 1004 individuos que conforman el grupo). Se encontraron seis tipos de diagnósticos presuntivos: ITU (infección de tracto urinario) 45,2\%, IRA (insuficiencia renal aguda) $20,2 \%$, urolitiasis $14,3 \%$, cistitis $10,7 \%$, pielonefritis $5 \%$ e IRC (insuficiencia renal crónica) 4,8\%.

El sistema cardiovascular estuvo afectado en 78 pacientes (3,2\%), la mayoría de más de 7 años 
de edad. Los diagnósticos presuntivos más frecuentes en este sistema fueron las valvulopatías (62,3\%) y la cardiomiopatía dilatada $(9,5 \%)$. Este sistema fue motivo de consulta en el $5 \%$ de los pacientes del Grupo D (mayores de 3 años). El sistema respiratorio estaba alterado en 179 pacientes (7,4\%). El 4\% de los pacientes con afección respiratoria tenía entre 13 y 36 meses de edad (Grupo c). Se encontraron 15 tipos de diagnósticos presuntivos en 145 perros. Se destacó la traqueobronquitis infecciosa $(57,9 \%)$, la bronquitis $(11,7 \%)$, la rinotraqueitis $(9 \%)$ y la neumonía $(7,6 \%)$.

El sistema óseo estuvo afectado en 182 pacientes $(6,71 \%), 119$ machos y 63 hembras. El $56 \%$ era del Grupo D, seguido del $23 \%$ del Grupo B. Se encontraron 14 tipos de diagnósticos presuntivos en 147 pacientes; con mayor frecuencia de fracturas traumáticas $(31,3 \%)$, displasia de cadera $(20,4 \%)$, lesiones discales (16,3\%) y enfermedad articular degenerativa (osteoartritis; 6,8\%).

Las afecciones musculares las tuvieron 141 pacientes $(5,8 \%)$, distribuidos en tres grupos de edad: 41 pacientes del Grupo B, 26 del Grupo cy 59 del Grupo D. Se encontraron nueve tipos de diagnósticos presuntivos en 177 pacientes. Tuvieron mayor frecuencia el traumatismo por golpe $(31,6 \%)$, las heridas por trauma $(20,9 \%)$, las heridas por mordedura $(18,1 \%)$, la inflamación de tejidos blandos $(11,9 \%)$ y el politraumatismo por atropellamiento $(10,7 \%)$.

El SNC (sistema nervioso central) y el SNP (sistema nervioso periférico) estuvieron afectados en 125 pacientes $(4,35 \%)$, y de ellos el $45 \%$ era del Grupo D. Se encontraron ocho tipos de diagnósticos presuntivos en 95 pacientes: la epilepsia fue la enfermedad más frecuente (59,3\%), seguida del trauma medular $(20,2 \%)$, la disfunción cognoscitiva (7,5\%), el síndrome vestibular (5,4\%), la intoxicación con medicamentos desconocidos $(2,6 \%)$, el trauma craneoencefálico $(2,5 \%)$, la hemivértebra $(1,3 \%)$ y la cauda equina $(0,9 \%)$.

Los problemas oftalmológicos los tuvieron 130 pacientes (4,56\% de toda la población). El $5 \%$ de esos pacientes era mayor de 3 años. Se encontraron 18 tipos de diagnósticos presuntivos en 127 pacientes. Las afecciones de mayor frecuencia fueron las úlceras corneales $(24,4 \%)$, la conjuntivitis (17,3\%), el ojo de cereza (11\%), los abscesos perioculares $(8,7 \%)$, las cataratas $(7,9 \%)$ y la queratoconjuntivitis seca $(7,5 \%)$. Los problemas de oído estuvieron presentes en 103 perros (3,81\%), entre los 12 y 36 meses de edad (Grupo c). El diagnóstico presuntivo más frecuente fue la otitis media $(87,4 \%)$.

El sistema reproductor estuvo afectado en 122 pacientes $(4,64 \%$ de la población total), con mayor frecuencia en los Grupos c y D $(7,8 \%$ y $6,7 \%$, respectivamente). Las 99 hembras $(81,1 \%)$ eran mayores de 2 años y 90 de ellas estaban sin esterilizar. Hubo 18,9\% de machos mayores de 2 años y solo tres estaban castrados. Se encontraron 19 tipos de diagnósticos presuntivos en 100 pacientes. En las hembras, los más representativos fueron: piómetra $(27 \%)$, parto distócico (24\%), vaginitis $(8 \%)$ y endometritis (6\%). En los machos (15), la patología más frecuente fue la prostatitis ( $9 \%)$.

El sistema endocrino fue el de menor frecuencia en motivos de consulta, con 17 pacientes $(0,4 \%)$, todos mayores de 2 años de edad. Se encontraron 13 tipos de diagnósticos presuntivos endocrinos/metabólicos en 116 caninos; sobresalen el hipotiroidismo (37,9\%), la deficiencia nutricional/vitamínica inespecífica $(22,4 \%)$ y la diabetes mellitus $(7,1 \%)$.

En cuanto a las enfermedades infecciosas, se encontraron ocho tipos de diagnósticos presuntivos en 93 perros afectados. Se destacan el moquillo canino (38,7\%), el parvovirus canino $(30,1 \%)$, los memoparásitos no específicos $(15,1 \%)$ y la ehrlichiosis $(9,6 \%)$. Las lesiones oncológicas se evidenciaron en 97 pacientes $(4,01 \%$ del total de la población), y se encontraron 18 tipos de diagnósticos presuntivos como neoplasia mamaria $(23,7 \%)$, neoplasias subcutáneas tipo lipoma $(21,6 \%)$, papilomatosis $(11,3 \%)$ y mastocitoma $(6,2 \%)$. Los pacientes tenían un promedio de 8 años.

Hubo 1,8\% de pacientes sanos cuyo motivo de consulta no obedecía a ninguna patología. Algunos solamente llegaban para revisión, vacunación y control de peso.

\section{Discusión}

La distribución de pacientes atendidos por mes no tuvo diferencias significativas. Se evidenció mayor concurrencia de pacientes en marzo $(17,69 \%)$ y el mes con menor concurrencia fue junio (11,68\%). Se debe insistir en la necesidad de hacer estos seguimientos epidemiológicos durante más de un año, debido a que en algunos meses, por diferentes factores de riesgo, puede haber un aumento de la prevalencia de algunas enfermedades, seguido de 
un aumento de la concurrencia de pacientes. Los cachorros son más susceptibles a las enfermedades digestivas, especialmente virales y parasitarias [7].

En otra investigación en Manizales, se encontraron datos similares [8]; los resultados obtenidos en este trabajo lo reafirman. Esto es debido a que el sistema inmune está subdesarrollado en cachorros y, en ocasiones, no tiene un control parasitario. Sin embargo, el cambio natural de fuente alimenticia debe considerarse [9]. Las dermatopatías son las afecciones más frecuentes seguidas de las enfermedades gastrointestinales. Un estudio retrospectivo acerca de patologías dermatológicas encontró que el 57,6\% de la población analizada tenía entre 1 y 7 años de edad [10]. En otra investigación, se afirmó que la mayoría de los problemas de piel se manifiesta entre los 0 y los 12 meses de edad [11].

También se ha encontrado que la piel ha sido el sistema más afectado en caninos, seguido del sistema digestivo [12]. Sin embargo, la influencia ambiental, las características de los diferentes sistemas sanitarios y las variaciones entre poblaciones deben tenerse en cuenta al momento de interpretar los datos. Además, la importancia estadística de estos reportes concluye que las enfermedades del sistema digestivo y dermatológico son las afecciones más frecuentes, sin tener diferencias estadísticas importantes entre ellas. Se debe considerar que al momento de la encuesta no se habían hecho pruebas complementarias para descartar la etiología de las lesiones.

En pacientes geriátricos, el envejecimiento natural altera la función renal, disminuyendo el flujo sanguíneo, la cantidad total de glomérulos funcionales y la tasa de filtración [13]. Las enfermedades renales en esta investigación se dieron sobre todo en el Grupo D. El sistema cardiovascular también se ve influido por la edad y por el sexo [14]. En el presente trabajo fue evidente, $y$ en pacientes geriátricos se pudo apreciar, que la enfermedad cardiovascular más común es la enfermedad mixomatosa valvular $(62,3 \%)$, lo que concuerda con datos epidemiológicos mundiales que informan cerca del $70 \%$ de prevalencia [15]. También se sabe que la enfermedad cardiovascular se da con más frecuencia en los machos y las enfermedades congénitas se dan con mayor frecuencia en las hembras [16].

En investigaciones anteriores, se ha encontrado cerca del $4 \%$ de prevalencia de alteraciones del sistema respiratorio [17], especialmente en pacientes entre 13 y 36 meses de edad. En nuestro estudio encontramos un 7,4\% de prevalencia. Posiblemente, las variabilidades agroecológicas de Manizales, la altitud (2160 msnm), el volcán Nevado del Ruiz y el clima son factores importantes para desencadenar afecciones respiratorias, lo que puede ser una explicación de la mayor presentación de este tipo de afecciones en caninos de la ciudad.

La displasia de cadera se presenta en el 20,4\% de los caninos, dato que coincide con los resultados de una investigación reciente en Portugal, en la que se observa una prevalencia del 23,8 \% [18]; es una de las afecciones ortopédicas más comunes de los caninos en el mundo, seguida de las fracturas traumáticas. Los motivos de consulta por afecciones musculares, nerviosas y endocrinas no tuvieron importancia significativa por grupo específico de edad, lo cual puede estar relacionado con el escaso número de pacientes en estos subgrupos y por el corto tiempo que duró el estudio. Debido a esto, no fue posible contrastar los datos con reportes epidemiológicos pasados. Las lesiones oftalmológicas se presentaron en 130 pacientes $(5,4 \%)$, y de manera similar, una investigación comunicó una incidencia del $5 \%$ en caninos mayores de 3 años de edad [10].

La anatomía y la fisiología del aparato reproductor de las hembras, la acción crónica de la progesterona y su influencia en el agrandamiento de la glándula mamaria las hace propensas a diferentes enfermedades a lo largo de su vida reproductiva [19, 20]. Este tipo de enfermedades se manifiestan en la edad adulta, y los datos obtenidos en este trabajo lo confirman; así mismo se dan principalmente en las hembras de mayor edad (81,2\%).

Los pacientes con neoplasias tenían un promedio de 8 años en esta investigación, se ha reportado que en caninos las neoplasias son más frecuentes entre los 6 y los 14 años de edad [21], lo que posiblemente se reafirma con nuestros hallazgos.

\section{Conclusiones}

La información facilitada en esta investigación proporciona una visión global del estado epidemiológico de Manizales; es importante tener en cuenta las limitaciones de la investigación, debido a que los datos fueron obtenidos a partir de caninos que acuden a consulta externa en clínicas veterinarias de Manizales. Solamente se consideran los hallazgos clínicos al momento de la llegada de cada paciente. Sin embargo, 
es evidente que la frecuencia de aparición de las diferentes afecciones concuerda con estudios epidemiológicos similares en otras ciudades del país y del mundo. Se recomienda que otras ciudades del país aporten datos de investigaciones semejantes.

\section{Referencias}

[1] Ramírez M. "My dog's just like me": dog ownership as a gender display. Symbolic Interaction. 2006;29:373-91.

[2] Power E. Furry families: making a human-dog family through home. Social \& Cultural Geography. 2008;9:535-55.

[3] Wells DL. Domestic dogs and human health: an overview. Brit J Health Psy. 2007;12:145-56.

[4] Flaquer L. Las políticas familiares en una perspectiva comparada. Barcelona; 2000.

[5] Gómez LF, Atehortúa CG, Orozco SC. La influencia de las mascotas en la vida humana. Rev Col Cien Pec. 2009;20:377-86.

[6] Friedmann E, Son H. The human companion animal bond: how humans benefit. Vet Clin North Am. 2009;39:293-326.

[7] Magne ML. Selected topics in Pediatric Gastroenterology. Vet Clin North Am Small Anim Pract. 2006; 36:533-48.

[8] Pedraza F, Ramírez G, Linares S. Frecuencia de presentación de enfermedades caninas diagnosticadas en el Hospital Veterinario de la Universidad de Caldas durante un año. Revista Veterinaria y Zootecnia de Caldas. 2004;13:42-8.

[9] Greco DS. Pediatric nutrition. Vet Clin North Am Small Anim Pract. 2014;44:265-73.

[10] Almansa JE, Galán J, Benavides O. Análisis retrospectivo de las historias clínicas de una clínica veterinaria en Bogotá. NOvA - Publicación Científica en Ciencias Biomédicas. 2007;8(5):168-76.

[11] Pulido-Villamarin A, Linares-Linares M., Castañeda-Salazar R., Gutiérrez-Granados C, Aranda-Silva M, Rueda-Varon M. A retrospective analysis (20092010) of the dermatological, ear and ophthalmologi- cal alterations with presumptive clinical diagnosis of mycosis in canines and felines. Universitas Scientiarum. 2011;16(3).

[12] Villegas SH, Duque CPT, Chavarriaga CMY, Suárez AU. Análisis retrospectivo de los registros clínicos del Centro de Veterinaria y Zootecnia de la Universidad CEs 2004-2009. CEs Med Vet Zoo. 2010;5:61-8.

[13] Hoffmann U, Sieber C. Options for renal replacement therapy in geriatric patients. Dtsch Med Wochenschr. 2014;139:1568-71.

[14] Borgarelli M, Haggstrom J. Canine degenerative myxomatous mitral valve disease: natural history, clinical presentation and therapy. Vet Clin North Am Small Anim Pract. 2010;40:651-63.

[15] Atkins C, Bonagura J, Ettinger S, Fox P, Gordon S, Haggstrom J, Hamlin R, Keene B, Luis-Fuentes V, Stepien R. Guidelines for the diagnosis and treatment of canine chronic valvular heart disease. J Vet Intern Med. 2009;23:1142-50.

[16] Bussadori C, Pradelli D, Borgarelli M, Chiavegato D, D’Agnolo G, Menegazzo L, Migliorini F, Santilli R, Zani A, Quintavalla C. Congenital heart disease in boxer dogs: results of 6 years of breed screening. The Veterinary Journal. 2009;181:187-92.

[17] Ramírez H, Calle E, Echevarría C, Morales C. Prevalencia de brucelosis canina en dos distritos de la Provincia Constitucional del Callao. Rev Inv Vet Perú. 2006;17:39-43.

[18] Ginja M, Silvestre A, Gonzalo-Orden J, Ferreira A. Diagnosis, genetic control and preventive management of canine hip dysplasia: a review. Vet J. 2010;184:269-76.

[19] Silva-Molano RF, Loaiza-Echeverri AM. Piometra en animales pequeños. Vet Zootec. 2007;1:71-86.

[20] Ortega-Pacheco A, Gutiérrez-Blanco E, Jiménez-Coello $\mathrm{M}$. Common lesions in the female reproductive tract of dogs and cats. Vet Clin North Am Small Anim Pract. 2012;42:547-59.

[21] Miller WH, Griffin CE, Campbell KL, Muller GH. Muller and Kirk's Small Animal Dermatology. Filadelfia: Elsevier Health Sciences; 2013. 